DOI: https://doi.org/10.11144/Javeriana.umed58-4.dmft

\title{
Guía colombiana de práctica clínica para el tratamiento de la diabetes mellitus tipo 2: falla al tratamiento inicial
}

\section{Colombian Clinical Practice Guideline for Treatment of Type 2 Diabetes Mellitus: First Line Treatment Failure}

\author{
Pablo Aschner M. \\ Hospital Universitario San Ignacio, Colombia \\ Óscar Mauricio Muñoz V.a \\ Hospital Universitario San Ignacio, Colombia \\ Diana Girón C. \\ Pontificia Universidad Javeriana, Colombia \\ Olga Milena García M. \\ Hospital Universitario San Ignacio, Colombia \\ Daniel Gerardo Fernández Á. \\ Hospital Universitario San Ignacio, Colombia \\ Luz Ángela Casas \\ Universidad del Valle, Colombia \\ Luisa Fernanda Bohórquez \\ Universidad Nacional de Colombia, Colombia \\ Clara María Arango T. \\ Universidad de Antioquia, Colombia
}

Fecha de recepción: 27/09/2016 | Fecha de aprobación: 18/01/2017

Liliana Carvajal

Asociación Colombiana de Diabetes, Colombia

Doris Amanda Ramírez

Universidad Nacional de Colombia, Colombia

Juan Guillermo Sarmiento

Hospital Universitario San Ignacio, Colombia

Cristian Alejandro Colon

Hospital Universitario San Ignacio, Colombia

Néstor Fabián Correa G.

Pontificia Universidad Javeriana, Colombia

Pilar Alarcón R.

Pontificia Universidad Javeriana, Colombia

Álvaro Andrés Bustamante S.

Pontificia Universidad Javeriana, Colombia

a Autor de correspondencia. Correo electrónico:

o.munoz@javeriana.edu.co

Cómo citar: Aschner P, Muñoz OM, Girón D, García OM, Fernández DG, Casas LA, Bohórquez LF, Arango CM, Carvajal L, Ramírez DA, Sarmiento JG, Colón CA, Correa NF, Alarcón P, Bustamante ÁA. Guía colombiana de práctica clínica para el tratamiento de la diabetes mellitus tipo 2: falla al tratamiento inicial. Univ Med. 2017;58(4):1-11. doi: https://doi.org/10,11144/ Javeriana.umed58-4.dmft

\section{RESUMEN}

Introducción: Existen diferentes opciones de manejo para pacientes con diabetes mellitus tipo 2 (DMT2) que ya iniciaron tratamiento farmacológico con metformina y no han alcanzado metas de control glucémico. Resulta prioritario definir pautas para escoger la mejor opción en estos pacientes, así como en aquellos que no han tenido un control 
óptimo con la combinación de dos medicamentos. Objetivo: Definir cuál es antidiabético de elección, entre sulfonilureas, tiazolidinedionas, inhibidores de DPP-4, agonista del receptor de GLP-1 o insulina basal, como segunda y tercera líneas de manejo en pacientes con DMT2. Métodos: Se elaboró la guía de práctica clínica, siguiendo los lineamientos de la guía metodológica del Ministerio de Salud y Protección Social colombiano. Se revisó la evidencia disponible de forma sistemática y se formularon las recomendaciones utilizando la metodología GRADE. Conclusiones: En pacientes con DMT2 y falla terapéutica al manejo con metformina como monoterapia $(\mathrm{HbA} 1 \mathrm{C}>7 \%)$ se recomienda como primera opción adicionar un inhibidor DPP-4, como segunda opción adicionar inhibidor SGLT2 o sulfonilureas con bajo riesgo de hipoglucemia y como tercera opción agregar insulina basal a los pacientes que con la combinación de dos fármacos fallen en alcanzar su meta de $\mathrm{HbA1C}$. Si la falla terapéutica se asocia con un IMC persistentemente $\geq$ 30, se sugiere la adición de un agonista de GLP-1 por el potencial beneficio sobre la reducción de peso.

Palabras clave

diabetes mellitus tipo 2; metformina; insuficiencia del tratamiento; guías de práctica clínica.

Introduction: There are different options to treat type 2 diabetes (DMT2) patients who began treatment with metformin and have not reached therapeutic golds. It is imperative to define rules to choose the best option, in these patients, as in those who have not achieved an optimal control under combined therapy. Aim: To define the best option between sulfonylureas, thiazolidinediones, DPP4 inhibitors, GLP-1 agonist or basal insulin, as second or third line treatment, in patients with DMT2 who have not reached therapeutic golds with metformin or combined therapy. Methods: A clinical practice guide has been developed following the broad outline of the methodological guide from the Colombian Ministry of Health and Social Welfare, with the aim of systematically gathering scientific evidence and formulating recommendations using the GRADE (Grading of Recommendations Assessment, Development and Evaluation) methodology. Conclusions: In patients with DMT2 who did not reach their therapeutic goal with metformin as a monotherapy (Hb1Ac $<7 \%$ ), addition of a second oral antidiabetic medication is recommended. it is recommended as a first step to add a DPP-4 inhibitor. It is suggested to add a SGLT2 inhibitor or a sulfonylurea having low risk of hypoglycemia as acceptable options. It is suggested to add basal insulin as a third antidiabetic medication if the combination of two pharmacological treatments does not enable the patient to reach and maintain the HbA1c goal. It is suggested to add a GLP-1 agonist if therapeutic failure appears in patients who remain obese (BMI $\geq 30 \mathrm{~kg} / \mathrm{m}^{2}$ ), considering its potential to reduce weight.
}

Keywords type 2 diabetes mellitus; metformin; treatment failure; clinical practice guidelines.

\section{Introducción}

En pacientes con diabetes mellitus tipo 2 (DMT2) que ya iniciaron tratamiento farmacológico con metformina y no han alcanzado metas de control glucémico debe realizarse el cambio de monoterapia a terapia combinada [1]. De acuerdo con las recomendaciones de la Asociación Americana de Diabetes (ADA) [2], si la meta establecida de hemoglobina glusosilada (HbA1c) no se logra después de tres meses una vez instaurado el tratamiento con metformina asociado con intervenciones en el estilo de vida, debe considerarse adicionar un segundo hipoglucemiante, dentro de los cuales se deben considerar: sulfonilureas, tiazolidinedionas, inhibidores de dipeptidil peptidasa-4 (DPP-4), agonista del receptor del péptido 1 relacionado con el glucagón (GLP-1) o insulina basal.

En promedio, un segundo agente se asocia con una reducción aproximada de entre 0,9\% y $1,1 \%$ en la HbA1c [3,4]. El hipoglucemiante de elección como antidiabético oral adicionado a la terapia debe hacerse según la capacidad de disminuir las concentraciones de glucosa y también con la capacidad de reducir el riesgo de complicaciones micro y macrovasculares [2,5]. Cada una de las diferentes opciones terapéuticas tiene ventajas y desventajas. Las sulfonilureas tienen alta eficacia para reducción de la hemoglobina glicosilada pero están asociadas con aumento de peso y riesgo moderado de hipoglucemia. Las tiazolidinedionas tienen una alta eficacia para la reducción de la hemoglobina glicosilada e incluso pueden tener un efecto más duradero respecto a la metformina y las sulfonilureas [6], con bajo riesgo de hipoglucemia, pero se asocian a ganancia de peso y efectos adversos como edema por retención de líquidos, falla cardiaca y fracturas óseas, con un alto costo $[7,8]$. En los últimos años se han 
posicionado los iDPP-4 como una muy buena alternativa en términos de riesgo-beneficio.

En caso de encontrarse cantidades $\mathrm{HbA1c}$ después de asociar dos antidiabéticos (incluida la metformina), se debe indicar el uso de una tercera medicación. Las opciones incluidas en esta tercera línea terapéutica, de acuerdo con la ADA/EASD (European Association for the Study of Diabetes) y la AACE/ACE (American Association of Clinical Endocrinologists) incluyen las sulfonilureas, tiazolidinedionas, agonistas del receptor del péptido similar al glucagón 1 (GLP-1RA), iDPP-4 (en caso de no haberse utilizado como segunda línea), la insulina (protamina neutra de Hagedorn o NPH, por su sigla en inglés; glargina, y detemir) y la SGLT-2 (Sodium-Glucose Linked Transporter 2), y es característica la aprobación de todos ellos (sin preferencia) entendiendo así su similar efectividad para los objetivos de la terapia antidiabética.

Para la decisión acerca de esta tercera línea terapéutica la ADA/EASD y la AACE/ACE [9] han integrado como parte fundamental de las decisiones la individualización del paciente y lo concerniente al "perfil de riesgo" de las terapias y combinaciones; pero con consideraciones específicas con respecto a sus posibles efectos adversos. El uso rutinario y eficaz de la insulina basal como tercer medicamento en triple terapia la hace la opción inicial. Los análogos de la insulina se consolidan como una buena opción, por su reducción en el desarrollo de hipoglucemia, comparativamente con NPH (aunque con evidencia del incremento de peso para el caso de detemir, no así para glargina). Alternativamente, el uso de los GLP-1 tiene ventajas en cuanto a la reducción de peso y el papel de los SGLT2 está por definirse.

El Ministerio de Salud y Protección Social encargó a la Pontificia Universidad Javeriana y a la Alianza del Centro Nacional de Investigación en Evidencia y Tecnologías en Salud (Cinets), conformada adicionalmente por la Universidad Nacional de Colombia y la Universidad de Antioquia, el desarrollo de una Guía clínica sobre la prevención, detección temprana, diagnóstico, tratamiento y seguimiento de la diabetes mellitus en la población mayor de 18 años. El resultado de ese proceso, en lo referente al tratamiento de pacientes que fallaron a la terapia de primera línea, se presenta de forma resumida en el presente artículo. Documentos complementarios abordarán los temas de tamización y diagnóstico, tratamiento de primera línea y estrategias para la detección de complicaciones.

Cabe resaltar que en este proceso también participaron activamente la Asociación Colombiana de Endocrinología, así como la Asociación Colombiana de Diabetes, y el Cinets.

\section{Métodos}

El proceso de desarrollo de la guía se describe detalladamente en el Manual para desarrollo de guías de práctica clínica y en la actualización de dicha metodología (disponible en la página web del Ministerio de Salud y Protección Social: http ://gpc.minsalud.gov.co) [10].

El desarrollo de la guía partió de preguntas específicas seleccionadas por su importancia clínica; se dio prioridad a los temas en que se consideró aún existe una discusión activa en la literatura mundial y a aquellos en los que se detectó una variabilidad injustificada en las conductas realizadas por los profesionales de salud en Colombia. Cabe aclarar que aquellos temas ampliamente aceptados en la literatura mundial y no ameritan, por tanto, un nuevo análisis de la literatura científica y se aceptaron como premisas a la presente guía, que se presentan en el presente documento.

La metodología planteada garantiza una búsqueda sistemática de la evidencia científica (tanto revisiones sistemáticas de la literatura como estudios primarios). Así mismo, establece de forma clara los criterios para seleccionar la evidencia que se va a utilizar, evaluando cuidadosamente su calidad. La versión completa de la guía, en la que se presentan los resultados de todas estas evaluaciones, puede consultarse en la página web del Ministerio de Salud y Protección Social colombianos, así como en la página web de la Alianza Cinets. 
El grupo metodológico preparó un resumen de la evidencia disponible y lo presentó al panel completo durante las reuniones de generación de recomendaciones. Este panel incluyó representantes de diversas especialidades médicas (endocrinólogos, médicos internistas y médicos generales), así como otros profesionales de la salud (nutricionistas o terapeutas físicos). Cabe resaltar que durante todo el proceso se contó también con representantes de los pacientes, que aportaron activamente en la generación de recomendaciones. Todos los integrantes del panel presentaron abiertamente la declaración de sus conflictos de interés. Estos documentos están disponibles en la versión completa de la guía.

Durante las reuniones de generación de recomendaciones se siguió la metodología propuesta por el Grading of Recommendations Assessment, Development and Evaluation Working Group (GRADE), que permite tener en cuenta no solo la calidad de la evidencia disponible, sino consideraciones de costos, preferencias de los pacientes y la relación entre los beneficios y riesgos de las tecnologías de interés (pruebas, estrategias de manejo, intervenciones o medicamentos). Las recomendaciones presentadas muestran, por tanto, la fuerza de la recomendación (fuerte o débil), su dirección (a favor o en contra de la intervención), así como la calidad de la evidencia que la sustenta (muy baja, baja, moderada, alta o consenso de expertos).

La versión final de la guía fue evaluada por pares nacionales, seleccionados por el Ministerio de Salud y de la Protección Social colombiano, expertos tanto en diabetes mellitus tipo 2 como en metodología. El grupo desarrollador de la guía tuvo en cuenta sus aportes.

\section{Resultados}

Se condujo una búsqueda sistemática de la literatura de novo para identificar metanálisis o revisiones sistemáticas de la literatura, publicadas hasta junio de 2014, empleando tres bases de datos (Medline, Cochrane, CRD Database). En el proceso de búsqueda de información para todas las preguntas de manejo farmacológico se identificaron catorce estudios $(3,4,12-23)$ que hacen referencia a la adición de un segundo y tercer antidiabético oral a metformina cuando no se haya alcanzado un control glucémico adecuado.

Impacto de la adición de un segundo antidiabético oral al tratamiento con metformina en pacientes que no lograron el control glucémico con metformina como monoterapia

La revisión sistemática desarrollada por Phung [11] evalúa el impacto de un segundo medicamento antidiabético tras el fallo farmacológico a manejo con metformina. La adición de sulfonilureas demostró mejoría del control glucémico, con una disminución estadística y clínicamente significativa de la hemoglobina glicosilada (diferencia de medias: $-0,79 \%$; IC95 \%: $-0,68$ a $-0,90)$. A pesar de ello se reportó un aumento de peso en el grupo de sulfonilureas adicionada a metformina de $1,99 \mathrm{~kg}$ (IC95\%: 0,86-3,12) y se demostró un aumento significativo en el riesgo de hipoglucemia (definida como cualquier hipoglucemia o hipoglucemia severa), con un riesgo relativo (RR) de 2,63 (IC95 \%: 0,76-9,13).

En cuanto al desenlace "efectos adversos asociados a la terapia combinada de sulfonilureas adicionada a metformina", el metanálisis de Zhang y colaboradores [12] reportó un aumento de la incidencia de eventos adversos relacionados con el sistema nervioso, entre ellos mareos, ansiedad, insomnio, vértigo (RR: 1,27; IC95 $\%: 1,03-1,57)$, comparado con metfomina como monoterapia. Por el contrario, se encontró disminución estadísticamente significativa de los eventos gastrointestinales incluyendo diarrea, náuseas y vómito (RR: 0,75; IC95 \%: 0,67-0,84).

La evidencia acerca de la terapia combinada de inhibidores de DPP4 adicionada a metformina, comparado con este último medicamento como monoterapia, mostró una reducción de los valores de $\mathrm{HbA1c}$ de 0,78\% (IC95\%: 0,64 $\%-0,93 \%)$ y aumento en la probabilidad de 
alcanzar las metas de manejo de HbA1C (RR: 2,51\%; IC95 \%: 2,04-3,22). No se demostró un incremento del peso corporal (diferencias de medias: $-0,14 \mathrm{~kg}$; IC95 \%: $-0,94$ a 0,63 kg), y el riesgo de hipoglucemia no tuvo diferencias estadísticamente significativas (RR: 0,63; IC95 $\%: 0,26-1,71)$. Dos estudios, el EXAMINE [13] y el SAVOR TIMI-53 [14], nos aportan para evaluar el riesgo de mortalidad, relacionada con la asociación de inhibidores de DPP4 al manejo farmacológico previo. De acuerdo con ellos, no se encontraron diferencias estadísticamente significativas en el riesgo de mortalidad por cualquier causa (RR: 0,65; IC95 \%: 0,39-1,09), ni en el riesgo de mortalidad de origen cardiovascular (RR: 0,97; IC95\%: 0,85-1,11); además, el riesgo de síndrome coronario agudo no aumentó el RR (0,97) (IC95 \%: 0,87-1,08; p = $0,59)$. Con respecto a accidente cerebrovascular no se evidenciaron diferencias estadísticamente significativas (RR: 0,98; IC95 \%: 0,81-1,18; p = $0,80)$.

De acuerdo con la revisión sistemática desarrollada por Li y colaboradores [15] no se encontró aumento del riesgo de pancreatitis (RR: 1,06; IC95 \%: 0,46-2,45), para el tratamiento con inhibidores DPP-4 adicionadas a metformina.

La evidencia disponible [11] acerca de la adición de tiazolidinedionas al manejo de pacientes que no alcanzaron metas de manejo con metformina reportó una disminución clínica y estadísticamente significativa en los valores de HbA1c de -0,85 \% (IC95 \%: - 1,08\% a -0,66 $\%$ para los análisis comparativos indirectos. Con respecto a la probabilidad de alcanzar las metas de HbA1c comparado con el placebo, se demostró un incremento de la tasa de éxito para alcanzar la meta, esto es, un valor de RR de 2,71 (IC95 \%: 1,74-3,8) para los análisis comparativos. En cuanto al riesgo de aumento de peso, los estudios que administraron pioglitazona demostraron aumento de peso de 1,5 a 2,3 $\mathrm{kg}$ en promedio. El riesgo de hipoglucemia no tuvo cambios estadísticamente significativos al comparar con placebo (RR: 0,56; IC95 \%: 0,19-1,69).

Con respecto al riesgo de muerte, infarto agudo de miocardio, evento cerebrovascular y falla cardiaca, la evidencia presentada por Lincoff y colaboradores [16], en la cual el manejo farmacológico fue la adición de pioglitazona a metformina, reportó que no existen diferencias en la incidencia de muerte (HR: 0,92; IC95 \%: 0,76-1,11), infarto agudo de miocardio (HR: 0,81; IC95\%: 0,64-1,02) o ataque cerebrovascular (HR: 0,80; IC95\%: 0,62-1,04). Sin embargo, reportó un aumento significativo en la incidencia de falla cardiaca (HR: 1,41; IC95 \%: 1,14-1,76).

Se presenta la evidencia acerca de la terapia combinada inhibidores de SGLT-2 adicionado a metformina, de acuerdo con un metanálisis realizado por Zhang y colegas [17], en el cual se evaluó el tratamiento combinado con inhibidores de SGLT-2 (dapagliflozina, ipragliflozina, canagliflozina, tofogliflozina, empagliflozina y luseogliflozina) adicionada a la terapia con metformina. Este estudio reportó una disminución de la $\mathrm{HbA1c}$ entre las 12 y las 24 semanas de DM de $-0,47 \%$ $(-0,66$ a $-0,27 ; \mathrm{p}<0,00001)$; al año DM, de $-0,37 \%(-0,77$ a $-0,03 ; \mathrm{p}=0,07)$; y después de 2 años de DM, $-0,4 \%(-1,09$ a $-0,28 ; \mathrm{p}=$ $0,24)$. En cuanto al desenlace de peso, se reportó una disminución entre las 12 y las 24 semanas de $-2,28 \mathrm{~kg}(-2,67 \mathrm{a}-1,88)$; al año, de $-2,60 \mathrm{~kg}$ $(-3,17$ a $-2,03)$, y después de 2 años, de $-3,03$ $\mathrm{kg}(-3,90$ a $-2,16)$. Para los efectos adversos de los inhibidores SGLT-2, este estudio reportó que la presencia de náuseas fue del 4,5\%; de la diarrea, del $6 \%$; de la infección de vías urinarias, del $7 \%$, y sospecha de infecciones genitales, del $2 \%$.

Para la terapia combinada con agonistas GLP-1 más metformina, la evidencia disponible dada por el metanálisis de Zintzaras y colaboradores [18] no reportó un aumento o disminución del riesgo de hipoglucemia con resultados muy imprecisos (OR: 0,54; IC95 \%: (0,08-3,48). Para el control glucémico este metanálisis reportó beneficio de la terapia combinada con GLP-1 más metformina, que mostró una mayor probabilidad de beneficio.

Impacto de la adición de un tercer antidiabético al tratamiento en pacientes quienes no lograron el 
control glucémico con metformina y un segundo antidiabético

La revisión sistemática de Gross y colaboradores [4] reportó que la adición de insulina al tratamiento con metformina + glibenclamida, comparado con placebo + metformina + glibenclamida, disminuyó las concentraciones de HbA1c $-0,71 \%(-0,95$ a $-0,47)$, en cuanto al desenlace del peso se reportó un aumento de peso de 2,31 kg (0,13-4,48).

Con respecto a la adición de inhibidores DPP4 al tratamiento con metformina + glibenclamida, comparado con placebo + metformina + glibenclamida, se reportó una disminución de la HbA1c de $-0,89 \%(-1,11$ a $-0,67)$.

En cuanto a la adición de tiazolidinedionas al tratamiento con metformina + glibenclamida comparado con placebo + metformina + glibenclamida, se reportó una disminución de la HbA1c de $-1,15 \%(-1,35$ a $-0,95)$. Para el desenlace del peso, esta revisión reportó un aumento del peso corporal en los pacientes que recibieron tiazolidinedionas de $2,4 \mathrm{~kg}$ $(-1,65-6,45)$.

La evidencia encontrada de la revisión de Gross, acerca de la adición de agonistas GLP-1 al tratamiento con metformina + glibenclamida, comparada con placebo + metformina + glibenclamida, reportó una disminución de la HbA1c de $-1,04 \%(-1,24$ a $-0,85)$; en el caso de los agonistas GLP-1 se encontró una disminución en el peso corporal de $-1,4 \mathrm{~kg}$ $(-2,9-0,08)$.

Para evaluar los desenlaces críticos como mortalidad, complicaciones microvasculares (nefropatía, retinopatía o neuropatía), complicaciones macrovasculares (accidente cerebrovascular, infarto agudo de miocardio o enfermedad arterial periférica), abandono (adherencia) y el desenlace calificado como importante /calidad de vida), se requirió buscar información como evidencia indirecta.

Se obtuvo información del metanálisis de Loke [19], el cual evaluó los efectos cardiovasculares con el uso de tiazolidenedionas. Se encontró que el uso de rosiglitazona, comparado con pioglitazona tuvo un incremento estadísticamente significativo en el riesgo de presentar: infarto agudo de miocardio (15 estudios; OR: 1,16; IC95\%: 1,07-1,24; p < 0,$001 ; \mathrm{I} 2=46 \%$ ), falla cardiaca congestiva (8 estudios; OR: 1,22; IC95 \%: 1,14-1,31; p < 0,$001 ; \mathrm{I} 2=37 \%$ ) y muerte ( 8 estudios; OR: 1,14 ; IC95 \%: 1,09-1,20; $<<0,001 ;$ I2 = $0 \%$ ).

\section{Discusión}

De acuerdo con la evidencia disponible acerca del impacto de la adición de un segundo antidiabético oral al tratamiento con metformina en pacientes que no lograron el control glucémico con metformina como monoterapia, con respecto a la $\mathrm{HbA} 1 \mathrm{C}$, se evaluaron dos desenlaces de efectividad: uno corresponde a la disminución en porcentaje del valor de la $\mathrm{HbA} 1 \mathrm{c}$ de 0,79\% (diferencia de medias: $-0,79 \%$; IC95 $\%$ : $-0,90$ a $-0,68 \%)$; el otro hace referencia a la capacidad de alcanzar la meta de HbA1c inferior al 7 \% (RR: 2,56\%; IC95 \%: 1,99-3,28). Los medicamentos mencionados fueron igual de efectivos en alcanzar estos objetivos; pero con respecto al aumento de peso, terapias como las sulfonilureas, glinidas y tiazolinedionas se encontró un aumento comparado con los inhibidores de DPP4, en los cuales existe una reducción.

Los estudios revisados e incluidos respecto al efecto de un tercer antidiabético al tratamiento con metformina y un segundo antidiabético demuestran las similitudes en términos de eficacia de los análogos de la insulina, los agonistas GLP-1 y los SGLT-2 contra placebo, puntualmente en el alcance de las metas de $\mathrm{HbA1c}$ y el beneficio en relación con el desarrollo de complicaciones micro y macrovasculares en pacientes diabéticos, usándolos como tercera medicación. Existen diferencias en cuanto a su impacto en el peso, pues se confirmó ganancia en usuarios de insulina y la disminución de este con el uso de agonistas de GLP-1 (aproximadamente $1,6 \mathrm{~kg}$ ). Teniendo en cuenta la similitud en cuanto a eficacia de los diferentes medicamentos como tercer antidiabético, el clínico podría 
escoger cualquiera de las tres opciones, según las características individuales cada paciente, buscando la mejor opción en logro de adherencia, comorbilidades, uso de otros fármacos, entre otras.

\section{Conclusión}

La revisión de la evidencia con respecto a la terapia de segunda línea en pacientes con DMT2 sin respuesta al manejo con dosis altas de metformina mostró que las diferentes opciones terapéuticas (sulfonilureas, glinidas, tiazolinedionas, inhibidores de DPP4 e inhibidores de SGLT2), al ser combinadas con metfomina, muestran un adecuado perfil en términos de efectividad. La diferencia entre las terapias combinadas radica de forma primordial en el perfil de eventos adversos y la seguridad. La hipoglucemia -importante marcador de seguridad - fue un evento adverso reportado de forma predominante en las sulfonilureas, comparado con los inhibidores de DPP4 o las tiazolinedionas, en las que no se produce de manera significativa este evento.

Teniendo en cuenta las opciones farmacológicas disponibles en nuestro medio, se consideró como primera elección en terapia de segunda línea combinado con metfomina el uso de inhibidores de DPP4, teniendo en cuenta que este grupo farmacológico contaba con el mejor perfil de efectividad y seguridad.

Se tomó en consideración la evidencia que sugiere aumento del riesgo de falla cardiaca, riesgo de fracturas y de cáncer de vejiga asociado al uso de pioglitazona, por lo cual no se aconseja la utilización de este antidiabético. Para riesgo de hipoglucemia asociada a las sulfonilureas, estas mostraron aumento estadística y clínicamente significativo, mayor cuando la combinación incluye glibenclamida. Con respecto a los análogos de GLP1, estos mostraron tener un efecto importante en la reducción de los valores de HbA1c; sin embargo, no justifica el uso como terapia de primera línea, dado su costo. Se consideran una alternativa aceptable como adición al tratamiento con metformina en pacientes con obesidad (índice de masa corporal $>35$ ), que no han logrado un reducción de peso con el tratamiento farmacológico y los cambios en el estilo de vida.

De acuerdo con la revisión de la evidencia acerca del impacto de la adición de un tercer antidiabético al tratamiento en pacientes quienes no lograron el control glucémico con metformina y un segundo antidiabético, los resultados en cuanto a disminución de los niveles de HbA1c, encontramos que al comparar contra placebo, el uso de tiazolidinedionas contra insulina, en el contexto de pacientes diabéticos que reciben metformina y glibenclamida de base, las tiazolidenedionas disminuyen los niveles de HbA1c, comparado con insulina (diferencia estadísticamente significativa).

En el caso de los agonistas GLP-1 en pacientes diabéticos que reciben metformina y glibenclamida, también se encontró una menor disminución en los niveles de $\mathrm{HbA1c}$. Al comparar el uso de tiazolidinedionas frente a insulina, en el contexto de pacientes diabéticos que reciben metformina y glibenclamida de base, encontramos un aumento en el peso corporal en los pacientes que recibieron tiazolidinedionas, comparado con los que recibieron insulina. Para el caso de los agonistas GLP-1, se halló el efecto opuesto con una disminución en el peso corporal.

\section{Recomendaciones}

En pacientes con DMT2 y falla terapéutica al manejo con metformina como monoterapia (HbA1C > 7\%), se recomienda adicionar un segundo antidiabético oral. Recomendación fuerte a favor. Calidad de la evidencia: moderada

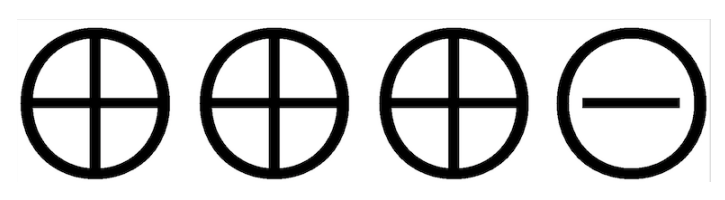

En pacientes con DMT2 y falla terapéutica al manejo con metformina 
como monoterapia (HbA1C > $7 \%$ ), se recomienda como primera opción adicionar un inhibidor DPP-4. Recomendación fuerte a favor. Calidad de la evidencia: moderada
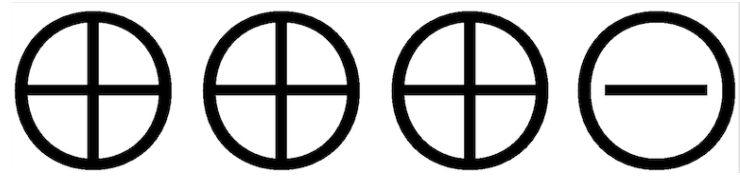

En pacientes con DMT2 y falla terapéutica al manejo con metformina como monoterapia (HbA1C $>7 \%$ ) se sugiere adicionar inhibidor SGLT2 si está contraindicado el iDPP-4. Recomendación débil a favor. Calidad de la evidencia: moderada
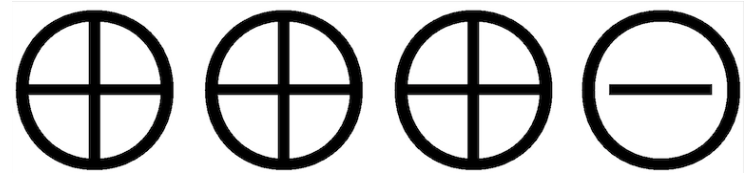

En pacientes con DMT2 y falla terapéutica al manejo con metformina como monoterapia $(\mathrm{HbA} 1 \mathrm{C}>7 \%)$, se sugiere agregar una sulfonilureas que tenga bajo riesgo de hipoglucemia (glimepirida o glicazida) cuando no estén disponibles o estén contraindicados los iDPP-4 y los iSGLT2. Recomendación débil a favor. Calidad de la evidencia: moderada
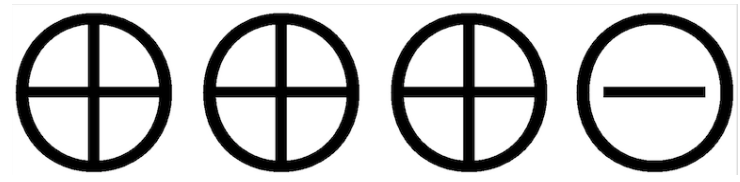

Los pacientes que requieran sulfonilureas deben recibir educación para prevenir, detectar y tratar oportunamente los episodios de hipoglicemia. Recomendación fuerte a favor. Consenso de expertos
En pacientes con DMT2 y falla terapéutica al manejo con metformina se sugiere $\mathrm{NO}$ adicionar glibenclamida por el alto riesgo de hipoglucemia. Recomendación débil en contra. Calidad de la evidencia: moderada
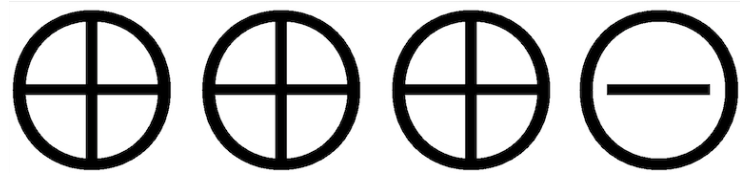

En pacientes con DMT2 y falla terapéutica al manejo con metformina se sugiere $\mathrm{NO}$ adicionar tiazolidinedionas por el mayor riesgo de edema, falla cardiaca y fracturas con esta terapia. Recomendación débil en contra. Calidad de la evidencia: moderada
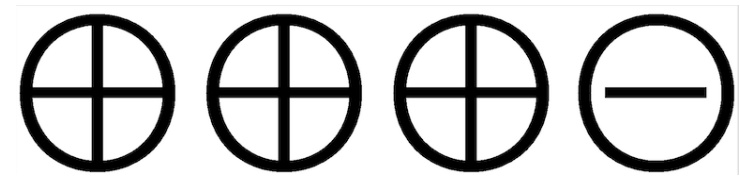

En pacientes con DMT2 y falla terapéutica al manejo con metformina que persistan con un $\mathrm{IMC} \geq 30$, se sugiere la adición de un agonista de GLP-1, por el potencial beneficio en la reducción de peso. Recomendación fuerte a favor. Calidad de la evidencia: moderada
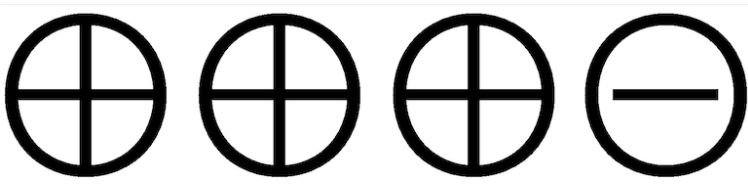

Se recomienda como tercera medicación antidiabética agregar insulina basal a los pacientes que con la combinación de dos fármacos fallen en alcanzar su meta de HbA1C y no estén obesos (índice de masa corporal < 30). Recomendación débil 
a favor. Calidad de la evidencia: moderada

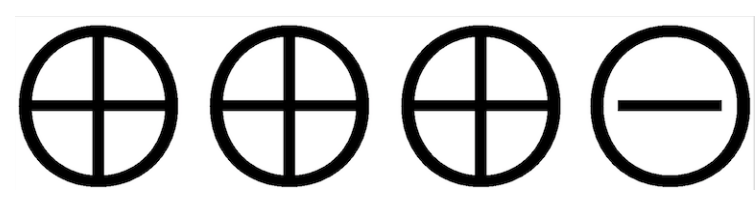

Se sugiere la adición de agonista de GLP-1 como tercera medicación antidiabética a los pacientes que fallen a la terapia combinada y persistan obesos (índice de masa corporal $\geq 30$ ). En caso de estar con un iDPP4, este se debe suspender. Recomendación débil a favor. Calidad de la evidencia: moderada

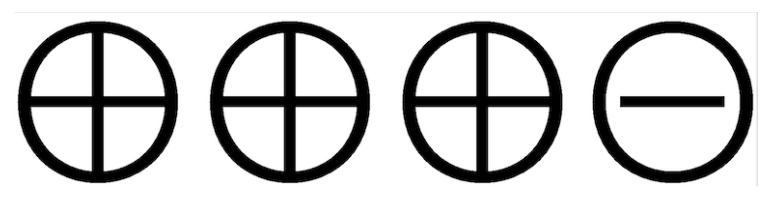

Se sugiere la adición de inhibidores de SGLT-2 en asociación a metformina. Recomendación débil a favor. Calidad de la evidencia: moderada

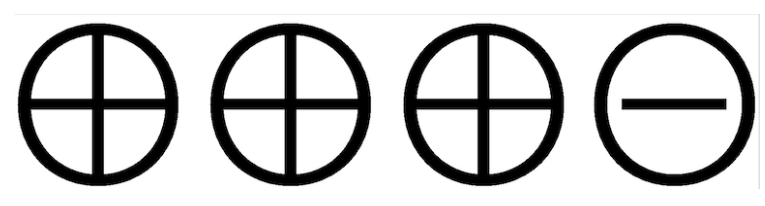

Se sugiere considerar la asociación de metformina y agonista de GLP-1 únicamente en los pacientes que no han alcanzado la meta terapéutica con metformina e inhibidores de SGLT-2 y que persistan obesos (índice de masa corporal $\geq 30$ ). Recomendación débil a favor. Consenso de expertos

\section{Puntos de buena práctica clínica}

Siempre que se detecte una falla en el logro de las metas terapéuticas con los fármacos, se deben revisar e intensificar también los cambios del estilo de vida hasta donde sea posible.

Con relación al peso corporal, se debe tener en cuenta que las sulfonilureas y las tiazolidinedionas lo incrementan, los inhibidores DPP4 no lo modifican, la metformina puede bajarlo y los inhibidores SGLT2 lo reducen al igual que los agonistas del receptor de GLP1.

Si se presentan hipoglucemias con la adición de sulfonilureas, se debe considerar el cambio a medicamentos que no causen hipoglucemia.

La adición de un agonista del receptor de GLP-1 requiere educar al paciente en el uso de la inyección subcutánea y la titulación de la dosis que debe incrementarse gradualmente para minimizar los efectos gastrointestinales como la náusea y el vómito.

Cuando el paciente se encuentra muy sintomático y clínicamente inestable, con pérdida aguda de peso, signos de deshidratación, evidencia de cetosis y glucemias muy elevadas, se recomienda agregar insulina.

La insulina NPH se puede utilizar como insulina basal cuando se inyecta a la hora de acostarse, hacia las 10:00-11:00 p. m.

Las insulinas análogas de acción prolongada (glargina, detemir o degludec) tienen menor riesgo de hipoglucemia cuando se comparan con la insulina NPH, por lo cual se prefieren cuando se desea que el paciente se mantenga en un control óptimo evitando hipoglucemias, o cuando estas se presentan al intentar alcanzar la meta de control glucémico. Estas insulinas se inyectan una vez al día a una hora fija, excepto el detemir, que puede administrarse dos veces al día si es necesario.

La dosis inicial de insulina basal es de 10 unidades/día o de 0,2 unidades $/ \mathrm{kg}$ de peso/día. Existen varios algoritmos para titularla, y todos se basan en incrementar la dosis en 2 a 4 unidades; a la vez, si la glucemia en ayunas está por encima del valor prefijado por dos a tres días seguidos o en el promedio de ese mismo intervalo.

Los agonistas del receptor de GLP-1 requieren una titulación progresiva para minimizar los efectos secundarios gastrointestinales y cada uno tiene sus instrucciones al respecto. Así mismo, cada uno tiene su propia forma de administración 
que puede ser una o dos veces al día, inclusive semanal.

Cuando no se logra la respuesta esperada con la insulina basal o con los agonistas del receptor GLP-1, se pueden combinar o utilizar esquemas de insulinoterapia intensiva. Sin embargo, en estos casos se recomienda que un equipo especializado reevalúe la meta y la necesidad de intensificar el tratamiento en cada paciente.

Durante la terapia con antidiabéticos orales que puedan causar hipoglucemia y con insulina, se debe educar al paciente en la detección temprana de los síntomas de hipoglucemia, así como en las acciones correctivas en caso de que se presente. Se debe poner especial cuidado en este sentido a la combinación de insulina con tratamientos que incluyan sulfonilureas. $\mathrm{Si}$ se presentan hipoglucemias y la insulina sigue siendo necesaria, se debe cambiar la sulfonilureas por otro medicamento que no cause hipoglucemia.

\section{Agradecimientos}

Fuente de financiación Ministerio de Salud y Protección Social. Alianza Cinets.

\section{Referencias}

1. Asociación Latinoamericana de Diabetes. Guías ALAD 2013 para el diagnóstico y manejo de la diabetes mellitus tipo 2 con medicina basada en evidencia. Revista de la ALAD [internet]. 2013. Disponible en: http://www.revistaalad.com/pdfs/Guias ALAD_11_Nov_2013.pdf

2. American Diabetes Association. Standards of medical care in diabetes--2013. Diabetes Care [Internet]. 2013 Jan [citado 2014 May 23];36 Suppl 1(October 2012):S11-66. Disponible en: http://www.pubmedcentral.nih.gov/arti clerender.fcgi? artid $=3537269 \&$ tool $=$ pmc entrez\&rendertype $=$ abstract

3. Bennett WL, Maruthur NM, Singh S, Segal JB, Wilson LM, et al. Comparative effectiveness and safety of medications for type 2 diabetes: an update including new drugs and 2-drug combinations. Ann Intern Med. 2011;154(9):602-13.

4. Gross JL, Kramer CK, Leita CB, et al. Effect of antihyperglycemic agents added to metformin and a sulfonylurea on glycemic control and weight gain in type 2 diabetes: a network meta-analysis. Ann Intern Med. 2011;154(10):672-9.

5. Canadian Diabetes Association Clinical Practice Guidelines Expert Committee, Cheng AY. Canadian Diabetes Asociation 2013 clinical practice guideline for the prevention and management of diabetes in Canada. Can J Diabetes. 2013;37 Suppl $1: S 1-3$.

6. Kahn SE, Haffner SM, Heise MA, Herman WH, Holman RR, Jones NP, et al. Glycemic durability of rosiglitazone, metformin, or glyburide monotherapy. N Engl J Med. 2006 Dec;355(23):2427-43.

7. Inzucchi SE, Bergenstal RM, Buse JB, Diamant M, Ferrannini E, Nauck M, et al. Management of hyperglycemia in type 2 diabetes: a patient-centered approach: position statement of the American Diabetes Association (ADA) and the European Association for the Study of Diabetes (EASD). Diabetes Care. 2012 Jun;35(6):1364-79.

8. Dormandy JA, Charbonnel B, Eckland DJA, Erdmann E, Massi-Benedetti M, Moules IK, et al. Secondary prevention of macrovascular events in patients with type 2 diabetes in the PROactive Study (PROspective pioglitAzone Clinical Trial In macroVascular Events): a randomised controlled trial. Lancet. 2005 Oct;366(9493):1279-89.

9. Handelsman Y, Mechanick JI, Dagogo-jack S, Davidson JA. AACE Guidelines. American Association of clinical endocrinologist medical guidelines for clinical practice for devoloping a diabetes mellitus comprehensive care plan. Diabetes Care. 2011;1-53.

10. Riethof M, Pl F, Lindvall B, Michels R, Connor OP, Redmon B, et al. Diagnosis and 
management of type 2 diabetes mellitus in adults. En: ICSI health care guideline. ICSI. 2012.

11. Phung OSJ. Effect of noninsulin antidiabetic drugs added to metformin therapy on glycemic control, weight gain, and hypoglycemia in type 2 diabetes. JAMA. 2014;303(14):1410-8.

12. Zhang F, Xiang H, Fan Y, Ganchuluun T-A, Kong W, Ouyang Q, et al. The effects of sulfonylureas plus metformin on lipids, blood pressure, and adverse events in type 2 diabetes: a meta-analysis of randomized controlled trials. Endocrine [Internet]. 2013 Dec [citado 2014 Sep 11];44(3):648-58. Disponible en: http://ww w.ncbi.nlm.nih.gov/pubmed/23657947

13. White WB, Cannon CP, Heller SR, Nissen SE, Bergenstal RM, Bakris GL, et al. Alogliptin after acute coronary syndrome in patients with type 2 diabetes. N Engl J Med. 2013 Oct;369(14):1327-35.

14. Monami M, Dicembrini I, Mannucci E. Dipeptidyl peptidase-4 inhibitors and heart failure: a meta-analysis of randomized clinical trials. Nutr Metab Cardiovasc Dis NMCD. 2014 Jul;24(7):689-97.

15. Li L, Shen J, Bala MM, Busse JW, Ebrahim $\mathrm{S}$, Vandvik PO, et al. Incretin treatment and risk of pancreatitis in patients with type 2 diabetes mellitus: systematic review and meta-analysis of randomised and nonrandomised studies. BMJ. 2014;348:g2366.

16. Lincoff A, Wolski K, Nicholls SJ, Nissen SE. Pioglitazone and risk of cardiovascular events in patients with type 2 diabetes mellitus. Jama. 2014;298(10):1180-8.

17. Zhang Q, Dou J, Lu J. Combinational therapy with metformin and sodium-glucose cotransporter inhibitors in management of type 2 diabetes: Systematic review and meta-analyses. Diabetes Res Clin Pract [Internet]. 2014 Sep [citado 2014 Oct 20];105(3):313-21. Disponible en: http://w ww.ncbi.nlm.nih.gov/pubmed/25015317

18. Zintzaras E, Miligkos M, Ziakas P, Balk EM, Mademtzoglou D, Doxani C, et al. Assessment of the relative effectiveness and tolerability of treatments of type 2 diabetes mellitus: a network meta-analysis. Clin Ther [Internet]. 2014 Aug 7 [citado 2014 Sep 3];1-11. Disponible en: http://ww w.ncbi.nlm.nih.gov/pubmed/25109773

19. Kong Y, Shing K. Comparative cardiovascular effects of thiazolidinediones: systematic review and meta-analysis of observational studies. BMJ [Internet]. 2011 Jan [citado 2014 Sep 11];342:d1309. Disponible en: http://www.pubmedcentral.nih.gov/arti clerender.fcgi? artid $=3230110$ \& tool $=$ pmc entrez\&rendertype $=$ abstract 